\title{
Auslander-Reiten Triangles and Grothendieck Groups of Triangulated Categories
}

\author{
Johanne Haugland ${ }^{1}$
}

Received: 7 April 2020 / Accepted: 26 May 2021 /Published online: 29 June 2021

(C) The Author(s) 2021

\begin{abstract}
We prove that if the Auslander-Reiten triangles generate the relations for the Grothendieck group of a Hom-finite Krull-Schmidt triangulated category with a (co)generator, then the category has only finitely many isomorphism classes of indecomposable objects up to translation. This gives a triangulated converse to a theorem of Butler and Auslander-Reiten on the relations for Grothendieck groups. Our approach has applications in the context of Frobenius categories.
\end{abstract}

Keywords Auslander-Reiten triangle · Grothendieck group · Triangulated category · Frobenius category

Mathematics Subject Classification (2010) Primary 18E30 - 18F30; Secondary 18E10 . $16 \mathrm{G} 70$

\section{Introduction}

The notion of almost split sequences was introduced by Auslander and Reiten in [4], and has played a fundamental role in the representation theory of finite dimensional algebras ever since [5]. The theory of almost split sequences, later called Auslander-Reiten sequences or just AR-sequences, has also greatly influenced other areas, such as algebraic geometry and algebraic topology $[2,14]$.

Happel defined Auslander-Reiten triangles in triangulated categories [11]. These play a similar role in the triangulated setting as AR-sequences do for abelian or exact categories. While it is known that AR-sequences always exist in the category of finitely generated modules over a finite dimensional algebra, the situation in the triangulated case turns out to be more complicated, and the associated bounded derived category will not necessarily have AR-triangles. In fact, Happel proved that this category has AR-triangles if and only

\footnotetext{
Presented by: Michela Varagnolo

Johanne Haugland

johanne.haugland@ntnu.no
}

1 Department of Mathematical Sciences, NTNU, NO-7491 Trondheim, Norway 
if the algebra is of finite global dimension [10,11]. Reiten and van den Bergh showed that a Hom-finite Krull-Schmidt triangulated category has AR-triangles if and only if it admits a Serre functor [18]. More recently, Diveris, Purin and Webb proved that if a category as above is connected and has a stable component of the Auslander-Reiten quiver of Dynkin tree class, then this implies existence of AR-triangles [8].

In the abelian setting, there is a well-studied relationship between AR-sequences, representation-finiteness and relations for the Grothendieck group. From Butler [7], Auslander-Reiten [3, Proposition 2.2] and Yoshino [21, Theorem 13.7], we know that if a complete Cohen-Macaulay local ring is of finite representation type, then the AuslanderReiten sequences generate the relations for the Grothendieck group of the category of Cohen-Macaulay modules. Here we say that our ring is of finite representation type if the category of Cohen-Macaulay modules has only finitely many isomorphism classes of indecomposable objects. A converse to this theorem is given by Auslander for artin algebras [1] and by Hiramatsu in the case of a Gorenstein ring with an isolated singularity [13, Theorem 1.2], where the latter is extended by Kobayashi [15, Theorem 1.2]. Results of the type described above were recently generalized to the setup of exact categories by Enomoto [9] and to certain extriangulated categories by Padrol, Palu, Pilaud and Plamondon [16].

A natural question to ask is whether there is a similar connection between AR-triangles, representation-finiteness and the relations for the Grothendieck group in the triangulated case. Xiao and Zhu give a partial answer to this question. Namely, they show that if our triangulated category is locally finite, then the AR-triangles generate the relations for the Grothendieck group [20, Theorem 2.1]. Beligiannis generalizes and gives a converse to this result for compactly generated triangulated categories [6, Theorem 12.1].

In this paper we consider the reverse direction of Xiao and Zhu from a different point of view. We prove that if the Auslander-Reiten triangles generate the relations for the Grothendieck group of a Hom-finite Krull-Schmidt triangulated category with a (co)generator, then the category has only finitely many isomorphism classes of indecomposable objects up to translation. We conclude by an application in the context of Frobenius categories. As an example, we see that our approach recovers results of Hiramatsu and Kobayashi for Gorenstein rings.

\section{Auslander-Reiten Triangles and Grothendieck Groups}

Let $R$ be a commutative ring. An $R$-linear category $\mathcal{T}$ is called Hom-finite provided that $\operatorname{Hom}_{\mathcal{T}}(X, Y)$ is of finite $R$-length for every pair of objects $X, Y$ in $\mathcal{T}$. An additive category is called a Krull-Schmidt category if every object can be written as a finite direct sum of indecomposable objects having local endomorphism rings. In a Krull-Schmidt category, it is well known that every object decomposes essentially uniquely in this way.

Throughout the rest of this paper, we let $\mathcal{T}$ be an essentially small $R$-linear triangulated category. We also assume that $\mathcal{T}$ is a Krull-Schmidt category which is Hom-finite over $R$. We let ind $(\mathcal{T})$ consist of the indecomposable objects of $\mathcal{T}$, while the translation functor of $\mathcal{T}$ is denoted by $\Sigma$. For simplicity, we use the notation $(A, B)=\operatorname{Hom}_{\mathcal{T}}(A, B)$ and $[A, B]=$ length $_{R}\left(\operatorname{Hom}_{\mathcal{T}}(A, B)\right)$.

We say that $\mathcal{T}$ has finitely many isomorphism classes of indecomposable objects up to translation if there is a finite subset of $\operatorname{ind}(\mathcal{T})$ such that for any $U \in \operatorname{ind}(\mathcal{T})$, there is an integer $n$ such that $\Sigma^{n} U$ is isomorphic to an object in our finite subset. 
Recall from [12] that a distinguished triangle $A \rightarrow B \stackrel{g}{\rightarrow} C \stackrel{h}{\rightarrow} \Sigma A$ in $\mathcal{T}$ is an Auslander-Reiten triangle if the following conditions are satisfied:

(1) $A, C \in \operatorname{ind}(\mathcal{T})$;

(2) $h \neq 0$;

(3) given any morphism $t: W \rightarrow C$ which is not a split-epimorphism, there is a morphism $t^{\prime}: W \rightarrow B$ such that $g \circ t^{\prime}=t$.

Let $\mathcal{F}(\mathcal{T})$ denote the free abelian group generated by all isomorphism classes $[A]$ of objects $A$ in $\mathcal{T}$, while $K_{0}(\mathcal{T}, 0)$ is the quotient of $\mathcal{F}(\mathcal{T})$ by the subgroup generated by the set $\{[A \oplus B]-[A]-[B] \mid A, B \in \mathcal{T}\}$. By abuse of notation, objects in $K_{0}(\mathcal{T}, 0)$ are also denoted by $[A]$. As $\mathcal{T}$ is a Krull-Schmidt category, the quotient $K_{0}(\mathcal{T}, 0)$ is isomorphic to the free abelian group generated by isomorphism classes of objects in $\operatorname{ind}(\mathcal{T})$.

Let $\operatorname{Ex}(\mathcal{T})$ be the subgroup of $K_{0}(\mathcal{T}, 0)$ generated by the subset

$$
\left\{\begin{array}{l|l}
{[X]-[Y]+[Z]} & \begin{array}{l}
\text { there exists a distinguished triangle } \\
X \rightarrow Y \rightarrow Z \rightarrow \Sigma X \text { in } \mathcal{T}
\end{array}
\end{array}\right\} .
$$

Similarly, we let $\operatorname{AR}(\mathcal{T})$ denote the subgroup of $K_{0}(\mathcal{T}, 0)$ generated by

$$
\left\{\begin{array}{l|l}
{[X]-[Y]+[Z]} & \begin{array}{l}
\text { there exists an AR-triangle } \\
X \rightarrow Y \rightarrow Z \rightarrow \Sigma X \text { in } \mathcal{T}
\end{array}
\end{array}\right\} .
$$

Recall from for instance [12] that the Grothendieck group of $\mathcal{T}$ is defined as $K_{0}(\mathcal{T})=K_{0}(\mathcal{T}, 0) / \operatorname{Ex}(\mathcal{T})$

In the proof of our main results, Theorem 2.4 and Theorem 2.5, we use the well-known fact that an equality in $K_{0}(\mathcal{T}, 0)$ can yield an equality in $\mathbb{Z}$. We need this in the case of $[U,-]$ and $[-, U]$ for an object $U$ in $\mathcal{T}$, but note that the following lemma could be phrased more generally in terms of additive functors.

Lemma 2.1 Suppose that $a_{1}\left[X_{1}\right]+\cdots+a_{r}\left[X_{r}\right]=0$ in $K_{0}(\mathcal{T}, 0)$ for integers $a_{i}$ and objects $X_{i}$ in $\mathcal{T}$. Then $a_{1}\left[U, X_{1}\right]+\cdots+a_{r}\left[U, X_{r}\right]=0$ and $a_{1}\left[X_{1}, U\right]+\cdots+a_{r}\left[X_{r}, U\right]=0$ in $\mathbb{Z}$ for any object $U$ in $\mathcal{T}$.

Proof Let $a_{1}\left[X_{1}\right]+\cdots+a_{r}\left[X_{r}\right]=0$ in $K_{0}(\mathcal{T}, 0)$. If $a_{i} \geq 0$ for every $i=1,2, \ldots, r$, we use the defining relations for $K_{0}(\mathcal{T}, 0)$ to obtain

$$
a_{1}\left[X_{1}\right]+\cdots+a_{r}\left[X_{r}\right]=\left[a_{1} X_{1} \oplus \cdots \oplus a_{r} X_{r}\right]=0,
$$

where $a_{i} X_{i}$ denotes the coproduct of the object $X_{i}$ with itself $a_{i}$ times. Consequently, the object $a_{1} X_{1} \oplus \cdots \oplus a_{r} X_{r}$ is zero in $\mathcal{T}$. Applying $[U,-]$ or $[-, U]$ and using additivity hence yields our desired equations.

If some of the coefficients $a_{i}$ are negative, we start by moving all negative terms to the right-hand side of our equality and proceed similarly.

The lemmas below, which yield a triangulated analogue of [15, Proposition 2.8], provide an important step in the proofs of Theorem 2.4 and Theorem 2.5. Note that parts of our proof of Lemma 2.2 is much the same as the proof of [8, Lemma 2.2]. Observe also that Lemma 2.3 follows from [19, Proposition 3.1] in the case where $R$ is an algebraically closed field, and that the argument generalizes to our context. We include complete proofs for the convenience of the reader. 
Lemma 2.2 Let $A \stackrel{f}{\rightarrow} B \stackrel{g}{\rightarrow} C \rightarrow \Sigma A$ be an AR-triangle in $\mathcal{T}$. The following statements hold for an object $U$ in $\mathcal{T}$ :

(1) The morphism $(U, B) \stackrel{g_{*}}{\rightarrow}(U, C)$ is surjective if and only if $C$ is not a direct summand in $U$.

(2) The morphism $(U, A) \stackrel{f_{*}}{\rightarrow}(U, B)$ is injective if and only if $\Sigma^{-1} C$ is not a direct summand in $U$.

(3) The morphism $(B, U) \stackrel{f^{*}}{\longrightarrow}(A, U)$ is surjective if and only if $A$ is not a direct summand in $U$.

(4) The morphism $(C, U) \stackrel{g^{*}}{\rightarrow}(B, U)$ is injective if and only if $\Sigma A$ is not a direct summand in $U$.

Proof Note that $C$ is a direct summand in $U$ if and only if there exists a split epimorphism $U \rightarrow C$. By the definition of an AR-triangle, this is equivalent to $g_{*}$ not being surjective, which proves (1).

Our triangle yields the long-exact sequence

$$
\cdots \rightarrow\left(U, \Sigma^{-1} B\right) \stackrel{\left(\Sigma^{-1} g\right)_{*}}{\longrightarrow}\left(U, \Sigma^{-1} C\right) \rightarrow(U, A) \stackrel{f_{*}}{\longrightarrow}(U, B) \rightarrow \cdots .
$$

The morphism $f_{*}$ is hence injective if and only if $\left(\Sigma^{-1} g\right)_{*}$ is surjective. By applying part (1) to the object $\Sigma U$, we see that $\left(\Sigma^{-1} g\right)_{*}$ is surjective if and only if $C$ is not a direct summand in $\Sigma U$, which is equivalent to $\Sigma^{-1} C$ not being a direct summand in $U$. This shows (2).

The statements (3) and (4) are verified dually, using that AR-triangles equivalently can be defined in terms of a factorization property for the leftmost morphism, see for instance [12].

Lemma 2.3 Let $A \stackrel{f}{\rightarrow} B \stackrel{g}{\rightarrow} C \rightarrow \Sigma A$ be an AR-triangle in $\mathcal{T}$. The following statements hold for an indecomposable object $U$ in $\mathcal{T}$ :

(1) We have $[U, A]-[U, B]+[U, C] \neq 0$ if and only if $U \simeq C$ or $U \simeq \Sigma^{-1} C$.

(2) We have $[A, U]-[B, U]+[C, U] \neq 0$ if and only if $U \simeq A$ or $U \simeq \Sigma A$.

Proof From the long exact Hom-sequence arising from our triangle, we get the exact sequence

$$
0 \rightarrow K \rightarrow(U, A) \stackrel{f_{*}}{\rightarrow}(U, B) \stackrel{g_{*}}{\rightarrow}(U, C) \rightarrow L \rightarrow 0,
$$

where $K=\operatorname{Ker}\left(f_{*}\right)$ and $L=\operatorname{Coker}\left(g_{*}\right)$. Splitting into short exact sequences and using our finiteness assumption, we see that the alternating sum of the lengths of the objects in the sequence vanishes. This gives the equation

$$
[U, A]-[U, B]+[U, C]=\operatorname{length}_{R}(K)+\operatorname{length}_{R}(L) .
$$

Consequently, we have $[U, A]-[U, B]+[U, C] \neq 0$ if and only if the right-hand side of the equation is also non-zero. This means that either $K$ or $L$ (or both) must be non-zero. The object $K$ is non-zero if and only if $f_{*}$ is not injective. By part Lemma 2.2 part (2), this is the case if and only if $\Sigma^{-1} C$ is a direct summand in $U$. Similarly, the object $L$ is non-zero if and only if $g_{*}$ is not surjective. Using part (1) of Lemma 2.2, this is equivalent to $C$ being a direct summand in $U$. As $U$ is indecomposable, a direct summand in $U$ is necessarily isomorphic to $U$, which finishes our proof of part (1).

Our second statement is shown dually, using part (3) and (4) of Lemma 2.2. 
We are now ready to prove our two main results, which show that we can study representation-finiteness of our category $\mathcal{T}$ by considering the relations for the associated Grothendieck group.

Theorem 2.4 Assume there is an object $X$ in $\mathcal{T}$ such that $\operatorname{Hom}_{\mathcal{T}}(Y, X) \neq 0$ or an object $X^{\prime}$ in $\mathcal{T}$ such that $\operatorname{Hom}_{\mathcal{T}}\left(X^{\prime}, Y\right) \neq 0$ for every non-zero $Y$ in $\mathcal{T}$. If $\operatorname{Ex}(\mathcal{T})=A R(\mathcal{T})$ in $K_{0}(\mathcal{T}, 0)$, then $\mathcal{T}$ has only finitely many isomorphism classes of indecomposable objects.

Proof Let $X$ be an object satisfying $\operatorname{Hom}_{\mathcal{T}}(Y, X) \neq 0$ for every nonzero $Y$ in $\mathcal{T}$, and consider the triangle $\Sigma^{-1} X \rightarrow 0 \rightarrow X \stackrel{1_{X}}{\rightarrow} X$. As this is a distinguished triangle, we have $\left[\Sigma^{-1} X\right]+[X] \in \operatorname{Ex}(\mathcal{T})$. By the assumption $\operatorname{Ex}(\mathcal{T})=\operatorname{AR}(\mathcal{T})$, there hence exist AR-triangles

$$
A_{i} \rightarrow B_{i} \rightarrow C_{i} \rightarrow \Sigma A_{i}
$$

and integers $a_{i}$ for $i=1,2, \ldots, r$ such that

$$
[X]+\left[\Sigma^{-1} X\right]=\sum_{i=1}^{r} a_{i}\left(\left[A_{i}\right]-\left[B_{i}\right]+\left[C_{i}\right]\right)
$$

in $K_{0}(\mathcal{T}, 0)$. Given an object $U$ in $\mathcal{T}$, Lemma 2.1 now yields the equality

$$
[U, X]+\left[U, \Sigma^{-1} X\right]=\sum_{i=1}^{r} a_{i}\left(\left[U, A_{i}\right]-\left[U, B_{i}\right]+\left[U, C_{i}\right]\right)
$$

in $\mathbb{Z}$. If $U$ is non-zero, our assumption on $X$ implies that the left-hand side of this equation is non-zero. Hence, there must for every non-zero object $U$ be an integer $i \in\{1, \ldots, r\}$ such that $\left[U, A_{i}\right]-\left[U, B_{i}\right]+\left[U, C_{i}\right] \neq 0$. In particular, this is true for every $U \in \operatorname{ind}(\mathcal{T})$. By Lemma 2.3 part (1), this means that any indecomposable object in $\mathcal{T}$ is isomorphic to an object in the finite set $\left\{C_{i}, \Sigma^{-1} C_{i}\right\}_{i=1}^{r}$, which yields our desired conclusion.

The proof in the dual case is similar, using Lemma 2.3 part (2).

In the theorem below, an object $X$ in $\mathcal{T}$ is called a generator of $\mathcal{T}$ if

$$
\operatorname{Hom}_{\mathcal{T}}^{*}(X, Y)=\bigoplus_{n \in \mathbb{Z}} \operatorname{Hom}_{\mathcal{T}}\left(X, \Sigma^{n} Y\right) \neq 0
$$

for any non-zero object $Y$ in $\mathcal{T}$. Dually, an object $X$ is called a cogenerator of $\mathcal{T}$ if $\operatorname{Hom}_{\mathcal{T}}^{*}(Y, X) \neq 0$ for any non-zero $Y$.

Theorem 2.5 Assume that $\mathcal{T}$ has a generator or a cogenerator. If $\operatorname{Ex}(\mathcal{T})=A R(\mathcal{T})$ in $K_{0}(\mathcal{T}, 0)$, then $\mathcal{T}$ has only finitely many isomorphism classes of indecomposable objects up to translation.

Proof Let $X$ be a cogenerator and consider an indecomposable object $U$ in $\mathcal{T}$. Notice that as $X$ is a cogenerator, there exists an integer $n$ such that $\operatorname{Hom}_{\mathcal{T}}\left(\Sigma^{n} U, X\right) \neq 0$. As in the proof of Theorem 2.4, our assumption $\operatorname{Ex}(\mathcal{T})=\operatorname{AR}(\mathcal{T})$ implies existence of a finite family of AR-triangles

$$
A_{i} \rightarrow B_{i} \rightarrow C_{i} \rightarrow \Sigma A_{i}
$$


which yields an equality

$$
\left[\Sigma^{n} U, X\right]+\left[\Sigma^{n} U, \Sigma^{-1} X\right]=\sum_{i=1}^{r} a_{i}\left(\left[\Sigma^{n} U, A_{i}\right]-\left[\Sigma^{n} U, B_{i}\right]+\left[\Sigma^{n} U, C_{i}\right]\right)
$$

in $\mathbb{Z}$. The left-hand side of this equation is non-zero, so there is an integer $i \in\{1, \ldots, r\}$ such that $\left[\Sigma^{n} U, A_{i}\right]-\left[\Sigma^{n} U, B_{i}\right]+\left[\Sigma^{n} U, C_{i}\right] \neq 0$. By applying Lemma 2.3 part (1), this yields that either $\Sigma^{n} U \simeq C_{i}$ or $\Sigma^{n+1} U \simeq C_{i}$. Consequently, every indecomposable object in $\mathcal{T}$ can be obtained as a translation of an object in the finite set $\left\{C_{i}\right\}_{i=1}^{r}$, which yields our desired conclusion.

The proof in the case where our category $\mathcal{T}$ has a generator is dual, using Lemma 2.3 part (2).

\section{Application to Frobenius Categories}

We now move on to an application of Theorem 2.4. Throughout the rest of the paper, let $\mathcal{C}$ be an essentially small $R$-linear Frobenius category. Recall that a Frobenius category is an exact category with enough projectives and injectives, and in which these two classes of objects coincide. The stable category of $\mathcal{C}$, i.e. the quotient category modulo projective objects, is denoted by $\underline{\mathcal{C}}$. We assume $\mathcal{C}$ to be a Krull-Schmidt category and that the stable category $\underline{\mathcal{C}}$ is Hom-finite.

As $\mathcal{C}$ is a Frobenius category, the associated stable category is triangulated. Recall that distinguished triangles in $\mathcal{C}$ are isomorphic to triangles of form $X \rightarrow Y \rightarrow Z \rightarrow \Omega^{-1} X$, where $0 \rightarrow X \rightarrow Y \rightarrow Z \rightarrow 0$ is a short exact sequence in $\mathcal{C}$ and $\Omega^{-1} X$ denotes the first cosyzygy of $X$. Note that $\Omega^{-1}$ is a well-defined autoequivalence on the stable category. The morphism $Z \rightarrow \Omega^{-1} X$ in our distinguished triangle above is obtained from the diagram

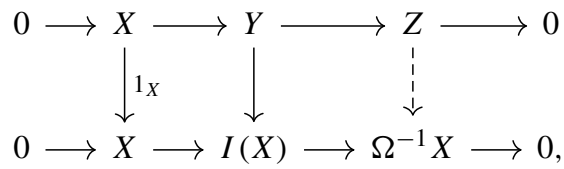

where $I(X)$ is injective and both rows are short exact sequences. For a more thorough introduction to exact categories and the stable category of a Frobenius category, see for instance [12].

Based on the correspondence between short exact sequences in a Frobenius category and distinguished triangles in its stable category, we get results also for Frobenius categories. In order to see this, we need to rephrase some of our terminology in the context of exact categories. Let us first recall that a short exact sequence $0 \rightarrow A \rightarrow B \stackrel{g}{\rightarrow} C \rightarrow 0$ in $\mathcal{C}$ is an Auslander-Reiten sequence if the following conditions are satisfied:

(1) $A, C \in \operatorname{ind}(\mathcal{C})$;

(2) the sequence does not split;

(3) given any morphism $t: W \rightarrow C$ which is not a split-epimorphism, there is a morphism $t^{\prime}: W \rightarrow B$ such that $g \circ t^{\prime}=t$.

As before, let $K_{0}(\mathcal{C}, 0)$ be the free abelian group generated by isomorphism classes of objects in $\mathcal{C}$ modulo the subgroup generated by $\{[\mathrm{A} \oplus B]-[A]-[B] \mid A, B \in \mathcal{C}\}$. Again, we can define the subgroups $\operatorname{Ex}(\mathcal{C})$ and $\operatorname{AR}(\mathcal{C})$ of $K_{0}(\mathcal{C}, 0)$, but now in terms of short 
exact sequences instead of distinguished triangles. Namely, we let $\operatorname{Ex}(\mathcal{C})$ be the subgroup generated by the subset

$$
\left\{\begin{array}{l|l}
{[X]-[Y]+[Z]} & \begin{array}{l}
\text { there exists a short exact sequence } \\
0 \rightarrow X \rightarrow Y \rightarrow Z \rightarrow 0 \text { in } \mathcal{C}
\end{array}
\end{array}\right\}
$$

and $\operatorname{AR}(\mathcal{C})$ the subgroup generated by

$$
\left\{\begin{array}{l|l}
{[X]-[Y]+[Z]} & \begin{array}{l}
\text { there exists an AR-sequence } \\
0 \rightarrow X \rightarrow Y \rightarrow Z \rightarrow 0 \text { in } \mathcal{C}
\end{array}
\end{array}\right\} .
$$

The next lemma describes a well-known correspondence between AR-sequences in $\mathcal{C}$ and AR-triangles in $\underline{\mathcal{C}}$, see [17, Lemma 3].

Lemma 3.1 An exact sequence $0 \rightarrow A \rightarrow B \rightarrow C \rightarrow 0$ in $\mathcal{C}$ is an $A R$-sequence in $\mathcal{C}$ if and only if the corresponding distinguished triangle $A \rightarrow B \rightarrow C \rightarrow \Omega^{-1} A$ in $\mathcal{C}$ is an AR-triangle in $\mathcal{C}$.

We are now ready to show the following lemma regarding the subgroups $\operatorname{Ex}(\mathcal{C})$ and $\mathrm{AR}(\mathcal{C})$ of $K_{0}(\mathcal{C}, 0)$ and the analogous subgroups of $K_{0}(\underline{\mathcal{C}}, 0)$.

Lemma 3.2 If $\operatorname{Ex}(\mathcal{C})=A R(\mathcal{C})$ in $K_{0}(\mathcal{C}, 0)$, then $\operatorname{Ex}(\underline{\mathcal{C}})=A R(\underline{\mathcal{C}})$ in $K_{0}(\underline{\mathcal{C}}, 0)$.

Proof Assume $\operatorname{Ex}(\mathcal{C})=\operatorname{AR}(\mathcal{C})$ in $K_{0}(\mathcal{C}, 0)$ and consider a distinguished triangle in $\underline{\mathcal{C}}$. As we work with isomorphism classes of objects, we can assume that our triangle is of the form $X \rightarrow Y \rightarrow Z \rightarrow \Omega^{-1} X$, where $0 \rightarrow X \rightarrow Y \rightarrow Z \rightarrow 0$ is a short exact sequence in $\mathcal{C}$. Since $\operatorname{Ex}(\mathcal{C})=\operatorname{AR}(\mathcal{C})$, there exist AR-sequences $0 \rightarrow A_{i} \rightarrow B_{i} \rightarrow C_{i} \rightarrow 0$ and integers $a_{i}$ for $i=1,2, \ldots, r$ such that

$$
[X]-[Y]+[Z]=\sum_{i=1}^{r} a_{i}\left(\left[A_{i}\right]-\left[B_{i}\right]+\left[C_{i}\right]\right)
$$

in $K_{0}(\mathcal{C}, 0)$, and hence also in $K_{0}(\underline{\mathcal{C}}, 0)$. By Lemma 3.1, the right-hand side of this equation is contained in $\operatorname{AR}(\underline{\mathcal{C}})$. Thus, we have shown that $\operatorname{Ex}(\underline{\mathcal{C}}) \subseteq \operatorname{AR}(\underline{\mathcal{C}})$. The reverse inclusion is clear.

We hence have the following corollary to Theorem 2.4 .

Corollary 3.3 Assume there is an object $X$ in $\mathcal{C}$ such that $\operatorname{Hom}_{\mathcal{C}}(Y, X) \neq 0$ or an object $X^{\prime}$ in $\mathcal{C}$ such that $\operatorname{Hom}_{\underline{\mathcal{C}}}\left(X^{\prime}, Y\right) \neq 0$ for every non-zero $Y$ in $\underline{\mathcal{C}}$. If $\bar{E} x(\mathcal{C})=A R(\mathcal{C})$ in $K_{0}(\mathcal{C}, 0)$, then the following statements hold:

(1) The category $\mathcal{C}$ has only finitely many isomorphism classes of non-projective indecomposable objects.

(2) If $\mathcal{C}$ has only finitely many indecomposable projective objects up to isomorphism, then $\mathcal{C}$ has only finitely many isomorphism classes of indecomposable objects.

Proof As $\mathcal{C}$ is an essentially small $R$-linear Krull-Schmidt category, the same is true for the stable category $\underline{\mathcal{C}}$. $\operatorname{As} \operatorname{Ex}(\mathcal{C})=\operatorname{AR}(\mathcal{C})$ in $K_{0}(\mathcal{C}, 0)$, Lemma 3.2 yields that $\operatorname{Ex}(\underline{\mathcal{C}})=\operatorname{AR}(\underline{\mathcal{C}})$ in $K_{0}(\underline{\mathcal{C}}, 0)$. The result now follows from Theorem 2.4. 
Let us consider the example where $R$ is a complete Gorenstein local ring with an isolated singularity. Recall that the category of Cohen-Macaulay $R$-modules is Frobenius. As $R$ is an isolated singularity, the associated stable category is Hom-finite, and completeness of $R$ yields the Krull-Schmidt property. By [13, Lemma 2.1], our category has an object which satisfies the assumption in the corollary above. Since $R$ is local, there are only finitely many isomorphism classes of indecomposable projective objects. Consequently, part (2) of Corollary 3.3 yields that if the AR-triangles generate the relations for the Grothendieck group of this category, then $R$ has only finitely many isomorphism classes of indecomposable Cohen-Macaulay modules. This recovers [13, Theorem 1.2] of Hiramatsu.

Note that one could, if preferred, state Theorem 2.4 and Corollary 3.3 in terms of taking the tensor product with $\mathbb{Q}$, as in the result of Kobayashi [15, Theorem 1.2]. Hence, also Kobayashi's conclusions are recovered from our approach in the case of a complete Gorenstein ring.

Acknowledgements The author would like to thank her supervisor Petter Andreas Bergh for helpful discussions and comments. She would also thank an anonymous referee for careful reading and suggestions which led to significant improvement of the paper.

Funding Open access funding provided by NTNU Norwegian University of Science and Technology (incl St. Olavs Hospital - Trondheim University Hospital).

Open Access This article is licensed under a Creative Commons Attribution 4.0 International License, which permits use, sharing, adaptation, distribution and reproduction in any medium or format, as long as you give appropriate credit to the original author(s) and the source, provide a link to the Creative Commons licence, and indicate if changes were made. The images or other third party material in this article are included in the article's Creative Commons licence, unless indicated otherwise in a credit line to the material. If material is not included in the article's Creative Commons licence and your intended use is not permitted by statutory regulation or exceeds the permitted use, you will need to obtain permission directly from the copyright holder. To view a copy of this licence, visit http://creativecommons.org/licenses/by/4.0/.

\section{References}

1. Auslander, M.: Relations for Grothendieck groups of Artin algebras. Proc. Amer. Math. Soc. 91(3), 336-340 (1984)

2. Auslander, M.: Almost split sequences and algebraic geometry. In: Representations of algebras (Durham, 1985), London Math. Soc. Lecture Note Ser., vol. 116, pp. 165-179. Cambridge University Press, Cambridge (1986)

3. Auslander, M., Reiten, I.: Grothendieck groups of algebras and orders. J. Pure Appl. Algebra 39(1-2), 1-51 (1986)

4. Auslander, M., Reiten, I.: Representation theory of Artin algebras. III. Almost split sequences. Comm. Algebra 3, 239-294 (1975)

5. Auslander, M., Reiten, I., Smalø, S.O.: Representation Theory of Artin Algebras, Cambridge Studies in Advanced Mathematics, vol. 36, p. xiv+423. Cambridge University Press, Cambridge (1995)

6. Beligiannis, A.: Auslander-Reiten triangles, Ziegler spectra and Gorenstein rings. $K$-Theory 32(1), 1-82 (2004)

7. Butler, M.C.R.: Grothendieck Groups and Almost Split Sequences. Integral Representations and Applications (Oberwolfach 1980). Lecture Notes in Math., vol. 882, pp. 357-368. Springer, Berlin-New York (1981)

8. Diveris, K., Purin, M., Webb, P.: Combinatorial restrictions on the tree class of the Auslander-Reiten quiver of a triangulated category. Math. Z. 282(1-2), 405-410 (2016)

9. Enomoto, H.: Relations for Grothendieck groups and representation-finiteness. J. Algebra 539, 152-176 (2019)

10. Happel, D.: Auslander-Reiten triangles in derived categories of finite-dimensional algebras. Proc. Amer. Math. Soc. 112(3), 641-648 (1991) 
11. Happel, D.: On the derived category of a finite-dimensional algebra. Comment. Math. Helv. 62(3), 339389 (1987)

12. Happel, D.: Triangulated categories in the representation theory of finite-dimensional algebras. London Mathematical Society Lecture Note Series, vol. 119, p. x+208. Cambridge University Press, Cambridge (1988)

13. Hiramatsu, N.: Relations for Grothendieck groups of Gorenstein rings. Proc. Amer. Math. Soc. 145(2), 559-562 (2017)

14. Jørgensen, P.: Calabi-Yau categories and Poincaré duality spaces. Trends in representation theory of algebras and related topics. EMS Ser. Congr. Rep., Eur. Math. Soc., Zürich, pp .399-431 (2008)

15. Kobayashi, T.: Syzygies of Cohen-Macaulay modules and Grothendieck groups. J. Algebra 490, 372379 (2017)

16. Padrol, A., Palu, Y., Pilaud, V., Plamondon, P.-G.: Associahedra for finite type cluster algebras and minimal relations between $g$-vectors, arXiv:1906.06861 (2019)

17. Roggenkamp, K.W.: Auslander-Reiten triangles in derived categories. Forum Math. 8(5), 509-533 (1996)

18. Reiten, I., Van den Bergh, M.: Noetherian hereditary abelian categories satisfying Serre duality. J. Amer. Math. Soc. 15(2), 295-366 (2002)

19. Webb, P.: Bilinear Forms on Grothendieck Groups of Triangulated Categories. Geometric and Topological Aspects of the Representation Theory of Finite Groups. Springer Proc. Math. Stat., vol. 242, pp. 465-480. Springer, Cham (2018)

20. Xiao, J., Zhu, B.: Relations for the Grothendieck groups of triangulated categories. J. Algebra 257(1), 37-50 (2002)

21. Yoshino, Y.: Cohen-Macaulay Modules Over Cohen-Macaulay Rings. London Mathematical Society Lecture Note Series, vol. 146, p. viii+177. Cambridge University Press, Cambridge (1990)

Publisher's Note Springer Nature remains neutral with regard to jurisdictional claims in published maps and institutional affiliations. 